\title{
1 Aniridia-related Keratopathy Relevant Cell Signaling Pathways \\ 2 in Human Fetal Corneas
}

3

4 André Vicente $^{\dagger 1}$, Marta Sloniecka ${ }^{\dagger 1}$, Berit Byström ${ }^{1}$, Jing-Xia Liu ${ }^{2}$, Fátima Pedrosa

5 Domellöf ${ }^{1,2 *}$

6

7 Department of Clinical Science, Ophthalmology, Umeå University, Umeå, Sweden;

$8 \quad{ }^{2}$ Department of Integrative Medical Biology, Section for Anatomy, Umeå University, Umeå,

9 Sweden;

10

$11 \dagger$ André Vicente and Marta Sloniecka are joint first authors

$13 *$ Correspondence to:

14 Fátima Pedrosa Domellöf

15 Department of Clinical Science, Ophthalmology

16 Umeå University

17 S-901 85 Umeå, Sweden

18 E-mail: fatima.pedrosa-domellof@umu.se

20 Running title: ARK relevant cell signaling pathways in human fetal corneas

22 Key words: aniridia, fetal cornea, adult cornea, sonic hedgehog, Notch, mTOR, Wnt

24 Funding: Swedish Research Council (2018-02401; Stockholm, Sweden), County Council of

25 Västerbotten in collaboration with Umeå University (Umeå, Sweden), Kronprinsessan

26 Margaretas Arbetsnämnd för Synskadade (Valdemarsvik, Sweden), The Medical Faculty, 
27 Umeå University (Umeå, Sweden), Ögonfonden (Stockholm, Sweden), Carmen and Bertil

28 Régners Stiftelsen (Stockholm, Sweden), and Åke Wibergs Stiftelse (Stockholm, Sweden)

29

30 Conflict of interest disclosure: The authors declare no competing or financial interests.

31

32

33

34

35

36

37

38

39

40

41

42

43

44

45

46

47

48

49

50

51 


\section{Abstract}

53 Background: To study aniridia-related keratopathy (ARK) relevant cell signaling pathways

54 (Notch1, Wnt/ß-catenin, Sonic hedgehog (SHH) and mTOR) in normal human fetal corneas 55 in comparison with normal human adult corneas.

56 Results: $20 \mathrm{wg}$ fetal and normal adult corneas showed similar staining patterns for Notch1,

57 however 10-11 wg fetal corneas showed increased presence of Notch1. Numb and Dlk1 had

58 an enhanced presence in the fetal corneas compared to the adult corneas. Fetal corneas

59 showed stronger immunolabeling with antibodies against $\beta$-catenin, Wnt5a and Wnt7a, Gli1,

60 Hes1, p-rpS6, and mTOR when compared to the adult corneas. Gene expression of Notch1,

61 Wnt5A, Wnt7A, $\beta$-catenin, Hes 1, mTOR and rps6 was higher in the 9-12 wg fetal corneas

62 when compared to adult corneas.

63 Conclusions: The cell signaling pathway differences found between human fetal and adult

64 corneas were similar to those previously found in ARK corneas with the exception of Notch1.

65 Analogous profiles of cell signaling pathway activation between human fetal corneas and

66 ARK corneas suggests that there is a less differentiated host milieu in ARK.

67 


\section{Introduction}

69

70 Aniridia is a congenital autosomal dominant disease caused by haploinsufficiency of the

71 PAX6 gene transcription factor. ${ }^{1}$ It includes, among other clinical features, aniridia-related

72 keratopathy (ARK), which classically occurs after childhood and recurs even if the patients

73 are submitted to corneal transplantation., 3 This chronic progressive keratopathy is

74 characterized by a multitude of defects such as disturbed corneal limbal cell differentiation,

75 fragile epithelial cells, compromised epithelial cell adhesion and a chronic wound-healing

76 state with compromised barrier function, which result in epithelial erosions, corneal

77 conjunctivalization and vascular pannus with significant impact on vision. ${ }^{4-6}$, We have

78 recently reported altered cell signaling pathways in ARK, with decreased detection of the

79 Notch1 cell signaling pathway and enhanced activation of the Sonic hedgehog (SHH), mTOR

80 and $\mathrm{Wnt} / \beta$-catenin cell signaling pathways not only in the epithelium but also in the

81 subepithelial pannus. ${ }^{7}$ Given that many tissues recapitulate patterns of fetal maturation during

82 regeneration, here we investigated whether these differences mimic the signaling pathway

83 patterns present during human fetal corneal development, which may reflect the presence of a

84 likewise immature host milieu in ARK corneas.

85 The development of the cornea includes two waves of neural crest cells and the primitive

86 corneal epithelium appears around day 33. The epithelium is then two-layered and already has

87 a basement membrane. ${ }^{8}$ At 7 weeks of gestation (wg), mesenchymal cells migrate to form the

88 corneal stroma and endothelium and at $7.5 \mathrm{wg}$ the keratoblasts, which will later differentiate

89 into keratocytes, are arranged in 4-5 incomplete layers with few collagen fibrils. The corneal

90 epithelium continues to evolve into a stratified squamous epithelium ${ }^{9}$ and by 11-12 $\mathrm{wg}$ all

91 corneal layers are formed, with the exception of the Bowman's layer. ${ }^{8}$ The epithelium has

92 then 2-3 cell layers and the stroma has 25-30 layers of keratoblasts and is separated from the 
93 endothelium by an irregular Descemet's membrane. ${ }^{10}$ The Bowman's layer is formed around 94 16-17 $\mathrm{wg}^{11}$ and by then the keratoblasts are distributed in a disorganized pattern in the 95 anterior stroma. ${ }^{10}$ At 7 months of gestation, the cornea already has an adult structure but in the 96 anterior stroma there are more keratoblasts and the collagen lamellae are still more randomly 97 oriented. ${ }^{8}$ The corneal structures generally develop adult features after the first two years of 98 life. ${ }^{9,12}$

99 Notch1, Wnt/ß-catenin, SHH, and mTOR cell signaling pathways are essential for eye 100 development, regulate both cell proliferation and homeostasis in mammals and are altered in 101 ARK corneas. ${ }^{7,13-16}$ The Notch1 signaling pathway coordinates corneal epithelial repair, 102 vertical migration and regulation of basal corneal epithelial cells. ${ }^{35}$ In the central corneal 103 epithelium, these processes depend on transient amplifying cells (TAC), which have high 104 migratory and proliferative capacity. ${ }^{17}$ TAC are derived from limbal epithelial stem cells 105 during normal corneal epithelial homeostasis and wound healing processes and migrate 106 centripetally in the basal layer to maintain the corneal epithelium. ${ }^{18}$ Dlk1, a transmembrane 107 protein that functions as negative regulator of Notch1, ensures that cells are kept in a 108 progenitor mode, which is essential in developmental processes. ${ }^{19}$ We have previously shown 109 that Dlk1 activation is increased in ARK corneal epithelial cells and stroma. ${ }^{7}$ Numb, another 110 important negative regulator of Notch1, is also upregulated in ARK corneas. ${ }^{7}$

111 Determination of limbal stem cell fate is regulated by the Notch1 and Wnt/ $\beta$-catenin signaling 112 pathways and are essential during normal tissue development and regeneration. ${ }^{16,}{ }^{20}$ The 113 extracellular ligands Wnt5a and Wnt7a stimulate the Wnt/ $\beta$-catenin signaling cascade, which 114 leads to proliferation of corneal limbal stem cells ${ }^{21}$, and is increased in ARK corneas. ${ }^{7} \beta$ 115 catenin is important for the regulation of epithelial differentiation and stratification. ${ }^{22}$

116 The SHH pathway regulates maintenance of cell polarity, cell differentiation and proliferation 117 and leads to activation of the glioma-associated oncogene homolog (Gli1) transcription factor 
118 that controls cell growth and survival. ${ }^{23}$ This pathway is upregulated in case of corneal

119 epithelium debridement ${ }^{24}$ and in ARK corneas. ${ }^{7}$ It shares a downstream effector, Hes1, with

120 the Notch 1 signaling pathway, which is essential for the regulation of corneal epithelial stem

121 cells and mammalian eye development ${ }^{25}$. The serine/threonine kinase mammalian target of

122 rapamycin (mTOR) signaling pathway regulates cell growth and proliferation. Activation of

123 the mTOR signaling pathway leads to phosphorylation of the ribosomal protein S6 (rpS6). ${ }^{26}$

124 mTOR signaling is upregulated in ARK corneas $^{7}$ and premature upregulation of mTORC1

125 during development in mice causes aniridia and anterior segment dysgenesis. ${ }^{15}$ 15, 16, 27

126 How these signaling pathways contribute to development of the human fetal cornea is

127 currently poorly understood. In the present study we employed immunostaining in

128 conjunction with fluorescence microscopy and qPCR to comparatively analyze potential

129 divergence of the important developmental signaling pathways Notch1, Wnt/ $\beta$-catenin, $\mathrm{SHH}$

130 and mTOR between fetal and adult human corneas.

132 Given that many tissues recapitulate patterns of fetal maturation during regeneration, here we

133 investigated whether these differences mimic the signaling pathway patterns present during

134 human fetal cornea development, which may reflect the presence of a likewise immature host

135 milieu in ARK corneas.

\section{2. Results}

139 Although the epithelium was not optimally preserved in the fetal corneas, it was still possible

140 to evaluate the staining patterns in all samples. The fetal corneas from 10-11 wg and $20 \mathrm{wg}$

141 and the adult corneas presented similar patterns of immunolabeling with the antibody against

142 Notch1, seen as streaks in the stroma and stronger staining around the basal layers of the 
143 corneal epithelium; however the 10-11 wg fetal cornea showed increased presence of Notch1

144 (fig. 1A-C). Dlk1 immunolabeling could be observed in the epithelial cells at both 10-11 wg 145 and $20 \mathrm{wg}$. Numb labelling was observed as streaks in the stroma of all fetal corneas. Numb 146 was more abundant in the anterior stroma (fig. 1D-E, asterisk). Conversely, Numb could not

147 be detected in the stroma of adult corneas but was present in the epithelial cells (fig. 1F).

148 Strong labeling with the Abs against Numb was observed in the epithelial cells of all fetal 149 corneas, clearly delineating their contours, and in stromal streaks (fig. 1G-H). In the $20 \mathrm{wg}$ 150 fetal corneas (fig. 1H) these Abs labelled the stroma in streaks more abundantly than in the 151 10-11 wg fetal corneas (fig. 1G). In contrast, in the adult corneas, Abs against Numb labelled 152 the epithelial cells but only sporadic streaks were present in the stroma (fig. 1I).

153 The epithelial cells of all fetal corneas were labelled with the Abs against Wnt5a (fig. 2A-B) 154 and the stroma was immunolabeled with these Abs in streaks, more abundantly in the 10-11 $155 \mathrm{wg}$ (fig. 2A) than in the $20 \mathrm{wg}$ fetal corneas (fig. 2B). In the $20 \mathrm{wg}$ fetal corneas the 156 immunolabeled streaks were slightly more abundant in the anterior stroma (fig. 2B). In 157 contrast, only the epithelium was labelled with the Abs against Wnt5a in the adult corneas and 158 the stroma was unlabeled (fig. 2C). Immunolabeling with Abs against Wnt7a was identified in 159 the epithelial cells and as streaks in the stroma of all fetal corneas (fig. 2D-E). Labelling in the 160 stroma of all fetal corneas presented as streaks which, as for Wnt5a, were slightly more 161 abundant in the 10-11wg (fig. 2D) than in $20 \mathrm{wg}$ corneas (fig. 2E). The Abs against Wnt7a 162 labeled the epithelium in adult corneas but in the stroma only extremely sparse streaks were 163 labelled (fig. 2F). The Ab against $\beta$-catenin intensely labelled the contours of the epithelial 164 cells in a similar pattern in both the 10-11 (fig. 2G) and $20 \mathrm{wg}$ (fig. $2 \mathrm{H}$ ) fetal corneas. In the 165 stroma of all fetal corneas, this $\mathrm{Ab}$ only labeled discrete streaks (fig. 2G-H). In the adult 166 corneas, labeling with the Ab against $\beta$-catenin was completely absent in the stroma, but the 167 epithelial cells were labeled in a pattern that was stronger in the basal region (fig. 2I). 
168 The Abs against Hes 1 strongly labelled the epithelium of all fetal corneas (fig. 3A-B). These

169 Abs labelled the stroma in streaks, more abundantly in the 10-11 wg (fig. 3A) than in the 20

170 wg fetal corneas (fig. 3B) and in the latter immunolabelling was more marked in the anterior

171 stroma (fig. 3B). These Abs did not label the epithelium or the stroma in the adult corneas

172 (fig. 3C). The epithelial cells in all fetal corneas were labeled with the Abs against Gli1 (fig.

173 3D-E) and the labelling was more abundant in the basal region of the epithelium in the $20 \mathrm{wg}$

174 fetal corneas (fig. 3E). Immunolabelling with these Abs was found in streaks in the stroma of

175 all fetal corneas (fig. 3D-E) and was stronger at $20 \mathrm{wg}$ (fig. 3E), whereas it was completely

176 absent in the epithelium and stroma of adult corneas (fig. 3F).

177 The Ab against mTOR labelled the epithelial cells in all fetal corneas (fig. 3G-H) but more

178 strongly in the $20 \mathrm{wg}$ fetal corneas (fig. $3 \mathrm{H}$ ). The stroma of all fetal corneas presented labelled

179 streaks (fig. 3G-H), which were more abundant in the 10-11 wg fetal corneas (fig. 3G),

180 whereas in the adult corneas no immunolabeling against mTOR was detected (fig. 3I).

181 Immunostaining against p-rpS6 was present in the epithelial cells and in abundant streaks in

182 the stroma of all fetal corneas, with similar intensity in both 10-11 wg and $20 \mathrm{wg}$ (fig. 3J-K).

183 In the adult corneas, labelling with the Ab against p-rpS6 was absent in the stroma and it was

184 only barely present in the surface of the epithelium, which could be indicative of non-specific

185 adherence to the epithelial surface (fig. 3L).

186 We subsequently examined the gene expression profiles of the cell signaling pathways by RT-

187 qPCR. The central part of the $9 \mathrm{wg}, 10-11 \mathrm{wg}$ fetal cornea, $12 \mathrm{wg}$ fetal cornea, and 3 adult

188 corneas were collected from frozen tissue sections using laser microdissection microscopy. In

189 comparison to the adult corneas, expression of Notch1 gene was 3.5 fold higher in 9-12 wg

190 fetal corneas (fig. 4A). Expression of Wnt5A, Wnt7A and $\beta$-catenin genes was increased by

$191 \quad$ 1.7, 3.75 and 2.14 fold, respectively (fig. 4B). Gene expression of Hes1, mTOR and rps6 was

192 increased by 2.2, 2.5 and 1.74 fold respectively (fig. 4C). 


\section{3. Discussion}

196 The present study is the first to investigate the patterns of immunolabeling and gene 197 expression of key cell signaling pathways related to cell proliferation and differentiation

198 (Notch1, Wnt/ß-catenin, SHH and mTOR) in the human fetal cornea. Cell differentiation is

199 altered in $\mathrm{ARK}^{5,6}$ and we have previously described changes in these cell signaling pathways

200 in naïve aniridia corneas and in failed corneal transplants with advanced ARK. ${ }^{7}$ The present

201 study allows a comparison between human fetal, normal adult and ARK corneas addressing

202 the question whether there is an immature host milieu in ARK that mimics that encountered 203 during fetal maturation.

204 The main limitation of this study is the reduced number of human corneal fetal samples 205 derived from the scarcity of opportunity to collect such samples. On the other hand, the fact 206 that the data was collected on human samples is a major strength of this study. We have

207 previously reported reduced immunolabeling detection of Notch1 and increased detection of 208 negative regulators of this signaling pathway (Dlk1 and Numb), in the epithelium and stromal 209 pannus of ARK corneas ${ }^{7}$ when compared to normal adult corneas. Notch1 is responsible for 210 corneal epithelial cell differentiation ${ }^{28}$ and lack of Notch1 in a mouse model leads to

211 keratinized hyperplastic, skin-like corneal epithelium with neovascularization in the stroma, ${ }^{29}$

212 which reproduces, in part, the changes encountered in ARK corneas. ${ }^{3}$ In contrast, in fetal 213 corneas, immunolabeling with Notch1 was detected in a similar pattern to that of normal adult 214 corneas with 10-11 wg fetal corneas showing slightly increased presence of Notch1. Notch1 215 gene expression analysis revealed that it is expressed more abundantly in the fetal corneas (fig 216 4A). Nevertheless, the negative regulators of Notch1, Dlk1 and Numb, were also present in 217 the stroma of the fetal corneas, as assessed by immunolabeling, but in the adult corneas they 
218 were only present in the epithelial cells. In the stroma of adult corneas, Dlk1 was absent and

219 Numb was only present in extremely sporadic streaks in a pattern that was opposite to that

220 observed in fetal and ARK corneas. This cell signaling pathway has a very diverse role in

221 development and in self-renewing tissues, such as the corneal epithelium, having functions

222 that range from serving as a gatekeeper for progenitor cells, cell lineage differentiation and

223 barrier function regulation ${ }^{28}$ and we have now described a stronger detection of the key

224 components of the Notch1 pathway in human fetal corneas during development. Dlk1 helps

225 cells remain immature and secures progenitor cell populations ${ }^{30}$ and is important in fetal

226 development. Here, we have revealed that it was abundantly expressed in the epithelial cells

227 and stroma of 10-11 wg and $20 \mathrm{wg}$ fetal corneas (fig. 1D-F) in a similar pattern to that

228 previously reported in ARK corneas. ${ }^{7}$ Numb, also a Notch1 negative regulator presented a

229 similar pattern of distribution to Dlk1 in the fetal corneas (fig 1G-I). There is a lack of data on

230 the possible roles of Numb and Dlk1 in the corneal stroma. Numb has been shown to

231 contribute to the maintenance of an undifferentiated state in epithelial progenitor cells in

232 epidermis $^{31}$ and the present findings can be interpreted as indicating the possibility of a

233 similar role in the human cornea.

234 The Wnt/ $\beta$-catenin cell signaling pathway has a decisive role in determining cell fate, 235 proliferation, differentiation and apoptosis during development, as well as maintenance of 236 stem cells and homeostasis in adult tissues ${ }^{32}$ and its presence is enhanced in the epithelium 237 and pannus of corneas with advanced ARK. ${ }^{7}$ In the present study we showed that in fetal 238 corneas at 10-11 wg and $20 \mathrm{wg}, \mathrm{Wnt} 5 \mathrm{a}$, and Wnt7a were abundantly identified in the corneal 239 epithelial cells and stroma, whereas their presence was practically limited to the epithelium in 240 normal adult corneas, as assessed by immunostaining. Upregulation of this signaling pathway 241 in fetal corneas (as shown by both immunolabelling and gene expression analysis), was 242 similar to what has been described in other ocular tissues during eye development. ${ }^{33,}{ }^{34} \mathrm{~A}$ 
243 previous study examined the importance of the $\mathrm{Wnt} / \beta$-catenin cell signaling pathway in

244 corneal development in mice and established that conditional ablation of $\beta$-catenin led to

245 precocious stratification of the corneal epithelium, postulating that this signaling pathway

246 plays a crucial role in epithelial stratification in corneal morphogenesis. ${ }^{22}$ Furthermore, it has

247 been shown that the $\mathrm{Wnt} / \beta$-catenin cell signaling pathway influences the development of

248 different ocular cell types in fetal corneas, including epithelial limbal stem cells, epithelial

249 cells $^{35,36}$ and keratocytes. ${ }^{22}$ In addition, it has been demonstrated before, in a mouse model,

250 that $\mathrm{Wnt} / \beta$-catenin cell signaling is not present in adult corneal limbus but that it is active in

251 wing and squamous corneal epithelium and in developing corneal stroma and endothelium, in

252 contrast to what happens in adult stroma and endothelium. ${ }^{16}$ Here we have shown that the

253 staining patterns regarding the Wnt/ß-catenin signaling pathway in the epithelium and stroma

254 of human fetal corneas seem to mimic a pattern of activation of this signaling pathway

255 described previously in mice. ${ }^{22}$ This signaling pathway seems to have a complex and

256 multifaceted role in the development and maintenance of the corneal epithelial cells. ${ }^{16}$

$257 \mathrm{SHH}$ is a secreted protein that works as a morphogen during development ${ }^{37}$ and directly

258 regulates the cell cycle, promoting proliferation by upregulating cyclin D1. ${ }^{38}$ Here we found a

259 stronger detection of elements of the SHH signaling pathway (Gli1 and Hes1) in 10-11 wg

260 and $20 \mathrm{wg}$ corneas, as well as increased Hes1 gene expression in 9-12 wg fetal corneas,

261 suggesting that activation of this pathway might be important during fetal corneal

262 development. Hes1, in particular, has a role in preventing differentiation and maintaining

263 progenitor cells and proliferation during development. ${ }^{25}$ Its abundant detection in the

264 epithelial cells and stroma of fetal corneas, compared with its absence in adult corneas,

265 suggests a role in cell proliferation during development and mimics the pattern described in

266 ARK corneas. ${ }^{7}$ SHH is upregulated in migrating corneal epithelial cells. ${ }^{24}$ Human mutations

267 in SHH result in holoprosencephaly which includes anophthalmia and cyclopia. ${ }^{39,} 40$ 
268 Furthermore, it has been proven that loss of SHH signaling in the lens disturbs the migration

269 of neural crest cells into the cornea. ${ }^{41}$ Therefore, SHH signaling has both direct and indirect

270 effects on corneal development. ${ }^{41}$ The results in the present study further emphasized its

271 importance during corneal fetal development and the important parallel with the patterns

272 encountered in ARK corneas. ${ }^{7}$

273 The mTOR cell signaling pathway can be described as a central regulator of cell proliferation,

274 growth, motility, transcription, protein synthesis, autophagy and survival. ${ }^{15,26}$ It regulates

275 apoptosis and cell proliferation in pterygium ${ }^{42}$ and was suggested to be upregulated in the

276 corneal epithelium and pannus in advanced ARK. ${ }^{7}$ In the present study, both mTOR and p-

$277 \mathrm{rpS6}$, a downstream element of this cell signaling pathway, were found in the fetal corneal

278 epithelial cells and stroma but were absent in normal adult corneas, suggesting that this

279 signaling pathway is also upregulated in fetal corneas at 10-11 wg and $20 \mathrm{wg}$, where cell

280 proliferation is most likely central. The gene expression analysis also showed that mTOR and

281 rps6 expression are higher in fetal corneas. The contribution of this signaling pathway to

282 proliferation has led to studies where its inhibition was used to treat corneal

283 neovascularization ${ }^{43}$ and transplant rejection. ${ }^{44}$ The effects of rapamycin, an inhibitor of the

284 mTOR signaling pathway, in human corneal epithelial cells in vitro have been previously

285 tested, indicating that it prevents the loss of corneal epithelial stem cells to replicative

286 senescence and apoptosis. ${ }^{45}$ Nevertheless, the effects of this signaling pathway on corneal

287 development are not known and should be further explored.

288 Our findings related to the immunolabeling pattern for these cell signaling pathways in the

289 basal layers of the corneal epithelium in fetal corneas, with labeling of areas usually

290 populated by TAC in adults, ${ }^{17}$ raised attention to the importance of epithelial basal cells with

291 high migratory and proliferative capacity in normal adults and ARK corneas. These cells are

292 formed by amplification of epithelial limbal stem cells and migrate centripetally during 
293 normal corneal epithelial homeostasis and wound healing in response to growth factors,

294 cytokines and changes in the extracellular matrix. ${ }^{17,18}$ The importance of these processes and

295 TAC in the chronic wound-healing in ARK remains a question to be further explored but

296 there is an apparent similarity between the regulation of cell signaling pathways related to the

297 activation of TAC in normal adults, epithelium in ARK corneas and in high proliferative fetal

298 epithelial cells.

299 The PAX6 gene is known to regulate transduction in ocular cells and to have a crucial role in

300 securing human corneal epithelium identity by regulating cell differentiation. ${ }^{46}$ We described

301 previously how Notch1, Wnt/ $\beta$-catenin, SHH and mTOR cell signaling pathways are altered

302 in patients with ARK, a condition resulting from reduced Pax6 protein levels. ${ }^{7}$ With the

303 exception of Notch1, the changes in the cell signaling pathways observed in the corneas of

304 patients with ARK and the patterns detected on human fetal corneas at 9-12 wg, 10-11 and 20

305 wg were analogous. This suggests that in corneas with advanced ARK, with reduced Pax6

306 protein levels, the cellular microenvironment mimics a less differentiated milieu, similar to

307 that occurring during normal fetal development in corneas with normal Pax6 protein levels.

308 In this study we analyzed localization and quantified the expression of Notch1, Wnt/ $\beta$ -

309 catenin, SHH and mTOR in normal human fetal corneas and healthy adult corneas. Cell

310 signaling in the human cornea during development is currently poorly understood but our data

311 has highlighted that certain pathways including mTOR1 and SHH may be essential in

312 differentiating epithelial cells. Similarity to what is present during normal corneal fetal

313 development, a more undifferentiated milieu, supports the proposed importance of host-

314 specific factors and the corneal microenvironment in the context of limbal stem cell

315 deficiency in ARK. We found that there are substantial similarities, excluding activation of

316 Notch1, between the gene expression profiles of key signaling components between fetal and

317 ARK corneas. 


\section{Experimental Procedures}

320

321

322

323

324

\subsection{Corneal Samples}

Eyes from eight human fetuses were collected, with ethical approval, after legal interruptions of pregnancy at $9 \mathrm{wg}(\mathrm{n}=2), 10-11 \mathrm{wg}(\mathrm{n}=2), 12 \mathrm{wg}(\mathrm{n}=2)$ and at $20 \mathrm{wg}(\mathrm{n}=2)$. Gestational age was calculated using the first day of the last menstrual period and confirmed with ultrasound. Five adult healthy corneas were collected from four male (74-, 76-, 82-, and 83-year-old) and one female (37-year-old) donors. These normal corneas were obtained from deceased individuals who, when alive, chose to donate their eyes post-mortem for transplantation and research purposes, according to Swedish law. The Regional Ethical Review Board in Umeå has determined the use of the post-mortem donated, anonymized tissue for study purpose to be exempt from the requirement for approval. The study followed the principles of the declaration of Helsinki.

The two human adult corneas (from 74- and 82-year-old males) used for immunochemistry were first fixed in formalin and embedded into paraffin wax. Superfrost ${ }^{\circledR}$ Plus slides were used to collect serial sections, $5 \mu \mathrm{m}$ thick, which were dried in a vertical position overnight at $60^{\circ} \mathrm{C}$ and then placed at $+4^{\circ} \mathrm{C}$ in a slide box, as previously described. ${ }^{7}$ The remaining three adult corneas and all fetal samples were mounted on cardboard with embedding medium (OCT cryomount, HistoLab Products AB, Gothenburg, Sweden), quickly frozen in propane cooled with liquid nitrogen and stored at $-80^{\circ} \mathrm{C}$. For immunohistochemistry $5 \mu \mathrm{m}$ thick serial sections were cut at $-23^{\circ} \mathrm{C}$ using a Leica CM3050 cryostat (Leica Microsystems, Wetzlar, Germany) and collected in Superfrost ${ }^{\circledR}$ Plus slides (Thermo Fisher Scientific, Rockford, IL, USA). For laser microdissections, $10 \mu \mathrm{m}$ thick serial sections of freshly frozen adult corneas, as well as $9 \mathrm{wg}, 10-11 \mathrm{wg}$ and $12 \mathrm{wg}$ fetal corneas were cut in RNase free conditions at $-23^{\circ} \mathrm{C}$ 
343 using a Leica CM3050 cryostat, and collected on NF1.0 PEN membrane slides (Zeiss, Jena,

344 Germany). Sections were fixed in ice cold $70 \%$ ethanol for 1 minute and transferred to $-80^{\circ} \mathrm{C}$

345 freezer until further processed.

346

$347 \quad 4.2$ Immunohistochemistry

348 The sections of the human fetal corneas were first brought to room temperature, left to dry (20

$349 \mathrm{~min}$ ) and then rinsed for three times $(5 \mathrm{~min})$ in $0,01 \mathrm{M}$ phosphate buffered saline (PBS).

350 Sections from adult control healthy corneas were de-waxed with Tissue-Clear® (1466, Sakura

351 Finetek Europe, Alphen aan den Rijn, Netherlands) and rehydrated. Antigen retrieval was

352 performed with a water bath at $95^{\circ} \mathrm{C}(30 \mathrm{~min})$ in pre-warmed citrate buffer (10mM Citric 353 acid, $0,05 \%$ Tween 20, $\mathrm{pH} 6,0$ ) or Tris-EDTA buffer (10mM Tris Base, 1mM EDTA, 0,05\%

354 Tween 20, $\mathrm{pH} 9.0)$. The sections were put at room temperature (20 $\mathrm{min})$ to cool down, 355 washed in running water (5 min) and then submerged in 0,01M PBS, three times (5 min).

356 In order to block unspecific binding, fetal and adult corneal sections were incubated with 5\%

357 normal goat or donkey serum at room temperature $(15 \mathrm{~min})$. Thereafter, the sections were

358 incubated with either monoclonal or polyclonal primary antibodies (Abs) specific against 359 components of the Notch1 (Notch1; Dlk1; Numb), Wnt/ $\beta$-catenin (Wnt5a; Wnt7a; $\beta$-catenin),

360 Sonic Hedgehog (Gli1; Hes1) and mTOR (mTOR1; p-rpS6) signaling pathways (Table 1), at

$361+4^{\circ} \mathrm{C}$ overnight. The sections were subsequently incubated with the adequate secondary

362 antibody (Table 2 ) at $37^{\circ} \mathrm{C}$ for $30 \mathrm{~min}$, submerged in $0,01 \mathrm{M}$ PBS for three times (5 min) and

363 then mounted with Vectashield ${ }^{\circledR}$ mounting media with 4',6-diamidino-2-phenylindole

364 (DAPI; H-1000 and H-1200; Vector laboratories, Inc, Burlingame, USA). Potential unspecific

365 binding was evaluated by omission of the primary antibody in control sections that were

366 otherwise processed similarly. 
367 We have previously worked with both freshly frozen and formalin fixed corneal specimens,

368 using the antibodies included in the present study. We have previously ascertained that the

369 staining pattern was similar irrespective of the tissue being freshly frozen or paraffin

370 embedded. $3,12,47,48$

371

$372 \quad 4.3$ Image acquisition

373 The sections were photographed using a Leica DM 6000 B microscope (Leica Microsystems,

374 Wetzlar, Germany), equipped with a digital high-speed fluorescence charge-coupled device

375 camera (Leica DFC360 FX; Leica Microsystems, Wetzlar, Germany). Image processing was

376 performed using Adobe Photoshop CS6 software (Adobe Systems, Inc., San Jose, CA, USA).

377 Both the immunostaining procedures and image acquisition with fluorescence microscopy

378 were performed using identical settings for all samples. Therefore, relative comparisons

379 between different samples treated with the same antibody could be performed.

380

\section{$381 \quad$ 4.4 Laser microdissections}

382 Before laser microdissection (LMD) was performed, the NF1.0 PEN membrane slides with 9

$383 \mathrm{wg}, 10-11 \mathrm{wg}$ and $12 \mathrm{wg}$ fetal corneas, and adult control corneas were transferred from $-80^{\circ} \mathrm{C}$

384 directly to ice cold $70 \%$ ethanol for 2 minutes, washed in nuclease free water in order to

385 remove residual OCT, and then dehydrated in 70, 96 and 100\% ethanol for 20 seconds each.

386 One additional wash in $100 \%$ ethanol was performed for $1 \mathrm{~min}$. Afterwards, the slides were

387 left to dry inside a fume hood for 10 minutes and transferred to a desiccator. LMD was

388 performed using a PALM MicroBeam microscope (Carl Zeiss Microscopy, Jena, Germany).

389 The central part of the corneas (stroma and epithelium) was carefully dissected, pooled (8

390 sections from each fetal cornea, and 16 sections from adult corneas), lysed in lysis buffer and

391 used for gene expression analysis. 


\section{$393 \quad 4.5$ RT-qPCR}

394 mRNA was extracted from the dissected corneas using RNA extraction kit (Qiagen, Venlo,

395 Netherlands) according to the manufacturer's instructions. Next, 35ng of RNA was reverse

396 transcribed into cDNA using the high-capacity cDNA reverse transcription kit (Thermo

397 Fisher). Notch1 (NOTCH1; Hs01062014_m1), Wnt5A (WNT5A; Hs00998537_m1), Wnt7A

398 (WNT7A; Hs01114990_m1), $\beta$-catenin (CTNNB1; Hs00355045_m1), Hes1 (HES1;

399 Hs00172878_m1), mTOR (mTOR, Hs00234508_m1), and rpS6 (RPS6; Hs01058685_g1)

400 probes were used in order to determine gene expression (Thermo Fisher). Samples were run

401 in duplicates in ViiA 7 TM 7 Real-Time PCR System (Thermo Fisher). $\beta$-actin and 18S probes

402 served as endogenous controls (Thermo Fisher). Analysis was performed with ViiA 7

403 Software (Thermo Fisher). The fold change gene expression in the fetal corneas at $9 \mathrm{wg}, 10$ -

$40411 \mathrm{wg}$ and $12 \mathrm{wg}$ was pooled together and compared to that of the adult corneas.

405

\section{$406 \quad 4.6$ Statistical analysis}

407 Data are presented as mean \pm SD. Statistical analysis was performed with unpaired t-test. A p-

408 value of $<0.05$ was considered statistically significant.

\section{$410 \quad$ References}

411 1. Lim HT, Kim DH, Kim H. PAX6 aniridia syndrome: clinics, genetics, and 412 therapeutics. Curr Opin Ophthalmol. Sep 2017;28(5):436-447.

413 doi:10.1097/ICU.0000000000000405

414 2. Bausili MM, Alvarez de Toledo J, Barraquer RI, Michael R, Tresserra F, de la Paz

415 MF. Histopathology Findings of Corneal Buttons in Congenital Aniridia Patients. Ophthalmic 416 Res. 2016;56(4):202-206. doi:10.1159/000444930

417 3. Vicente A, Bystrom B, Lindstrom M, Stenevi U, Pedrosa Domellof F. Aniridia-related 418 keratopathy: Structural changes in naive and transplanted corneal buttons. PLoS One.

419 2018;13(6):e0198822. doi:10.1371/journal.pone.0198822

420 4. Ou J, Walczysko P, Kucerova R, et al. Chronic wound state exacerbated by oxidative

421 stress in Pax6+/- aniridia-related keratopathy. J Pathol. Aug 2008;215(4):421-30.

422 doi:10.1002/path.2371 
423 5. Ramaesh K, Ramaesh T, Dutton GN, Dhillon B. Evolving concepts on the pathogenic 424 mechanisms of aniridia related keratopathy. Int J Biochem Cell Biol. Mar 2005;37(3):547-57. 425 doi:10.1016/j.biocel.2004.09.002

426 6. Latta L, Viestenz A, Stachon T, et al. Human aniridia limbal epithelial cells lack 427 expression of keratins K3 and K12. Exp Eye Res. Feb 2018;167:100-109.

428 doi:10.1016/j.exer.2017.11.005

429 7. Vicente A, Bystrom B, Pedrosa Domellof F. Altered Signaling Pathways in Aniridia-

430 Related Keratopathy. Invest Ophthalmol Vis Sci. Nov 1 2018;59(13):5531-5541.

431 doi:10.1167/iovs.18-25175

$4328 . \quad$ Remington LA, McGill EC. Clinical anatomy of the visual system. Butterworth433 Heinemann; 1998:ix, 258 p.

434 9. Herwig MC, Muller AM, Holz FG, Loeffler KU. Immunolocalization of different 435 collagens in the cornea of human fetal eyes: a developmental approach. Curr Eye Res. Jan 436 2013;38(1):60-9. doi:10.3109/02713683.2012.738461

437 10. Cook CS OV, Jakobiec FA. Prenatal development of the eye and its adnexa. In: 438 Tasman W JE, ed. Duane's foundations of clinical ophthalmology. Lippincott; 1994.

439 11. Wulle KG, Richter J. Electron microscopy of the early embryonic development of the 440 human corneal epithelium. Albrecht Von Graefes Arch Klin Exp Ophthalmol. Dec 8 $441 \quad 1978 ; 209(1): 39-49$.

442 12. Bystrom B, Virtanen I, Rousselle P, Gullberg D, Pedrosa-Domellof F. Distribution of 443 laminins in the developing human eye. Invest Ophthalmol Vis Sci. Mar 2006;47(3):777-85. 444 doi:10.1167/iovs.05-0367

445 13. Penton AL, Leonard LD, Spinner NB. Notch signaling in human development and 446 disease. Semin Cell Dev Biol. Jun 2012;23(4):450-7. doi:10.1016/j.semcdb.2012.01.010

447 14. Brennan D, Giles S. Sonic hedgehog expression is disrupted following in ovo ethanol 448 exposure during early chick eye development. Reprod Toxicol. Nov 2013;41:49-56. 449 doi:10.1016/j.reprotox.2013.05.012

450 15. Hagglund AC, Jones I, Carlsson L. A novel mouse model of anterior segment 451 dysgenesis (ASD): conditional deletion of Tsc1 disrupts ciliary body and iris development.

452 Dis Model Mech. Mar 1 2017;10(3):245-257. doi:10.1242/dmm.028605

453 16. Wang Y, Mahesh P, Wang Y, et al. Spatiotemporal dynamics of canonical Wnt 454 signaling during embryonic eye development and posterior capsular opacification (PCO). Exp 455 Eye Res. Jun 19 2018;175:148-158. doi:10.1016/j.exer.2018.06.020

456 17. Zhou MY, Li XM, Lavker RM. Transcriptional profiling of enriched Populations of 457 stem cells versus transient amplifying cells - A comparison of limbal and corneal epithelial 458 basal cells. Journal of Biological Chemistry. Jul 14 2006;281(28):19600-19609. 459 doi:10.1074/jbc.M600777200

460 18. Lehrer MS, Sun TT, Lavker RM. Strategies of epithelial repair: modulation of stem 461 cell and transit amplifying cell proliferation. J Cell Sci. Oct 1998;111 ( Pt 19):2867-75.

462 19. Falix FA, Aronson DC, Lamers WH, Gaemers IC. Possible roles of DLK1 in the 463 Notch pathway during development and disease. Biochim Biophys Acta. Jun 464 2012;1822(6):988-95. doi:10.1016/j.bbadis.2012.02.003

465 20. Tsai TH, Sun MH, Ho TC, Ma HI, Liu MY, Tsao YP. Notch prevents transforming 466 growth factor-beta-assisted epithelial-mesenchymal transition in cultured limbal progenitor 467 cells through the induction of Smad7. Mol Vis. 2014;20:522-34.

468 21. Nakatsu MN, Ding Z, Ng MY, Truong TT, Yu F, Deng SX. Wnt/beta-catenin 469 signaling regulates proliferation of human cornea epithelial stem/progenitor cells. Invest 470 Ophthalmol Vis Sci. Jul 1 2011;52(7):4734-41. doi:10.1167/iovs.10-6486 
471

472

473

474

475

476

477

478

479

480

481

482

483

484

485

486

487

488

489

490

491

492

493

494

495

496

497

498

499

500

501

502

503

504

505

506

507

508

509

510

511

512

513

514

515

516

517

518

519

22. Li G, Xu F, Zhu J, et al. Transcription Factor PAX6 (Paired Box 6) Controls Limbal Stem Cell Lineage in Development and Disease. J Biol Chem. Aug 14 2015;290(33):2044854. doi:10.1074/jbc.M115.662940

23. Taipale J, Beachy PA. The Hedgehog and Wnt signalling pathways in cancer. Nature. May 17 2001;411(6835):349-54. doi:10.1038/35077219

24. Saika S, Muragaki Y, Okada Y, et al. Sonic hedgehog expression and role in healing corneal epithelium. Invest Ophthalmol Vis Sci. Aug 2004;45(8):2577-85. doi:10.1167/iovs.040001

25. Lee HY, Wroblewski E, Philips GT, et al. Multiple requirements for Hes 1 during early eye formation. Dev Biol. Aug 15 2005;284(2):464-78. doi:10.1016/j.ydbio.2005.06.010 26. Dowling RJ, Topisirovic I, Fonseca BD, Sonenberg N. Dissecting the role of mTOR: lessons from mTOR inhibitors. Biochim Biophys Acta. Mar 2010;1804(3):433-9. doi:10.1016/j.bbapap.2009.12.001

27. Braune EB, Lendahl U. Notch -- a goldilocks signaling pathway in disease and cancer therapy. Discov Med. Mar 2016;21(115):189-96.

28. Ma A, Boulton M, Zhao B, Connon C, Cai J, Albon J. A role for notch signaling in human corneal epithelial cell differentiation and proliferation. Invest Ophthalmol Vis Sci. Aug 2007;48(8):3576-85. doi:10.1167/iovs.06-1373

29. Vauclair S, Majo F, Durham AD, Ghyselinck NB, Barrandon Y, Radtke F. Corneal epithelial cell fate is maintained during repair by Notch1 signaling via the regulation of vitamin A metabolism. Dev Cell. Aug 2007;13(2):242-53. doi:10.1016/j.devcel.2007.06.012 30. Abdallah BM, Jensen CH, Gutierrez G, Leslie RG, Jensen TG, Kassem M. Regulation of human skeletal stem cells differentiation by Dlk1/Pref-1. J Bone Miner Res. May 2004;19(5):841-52. doi:10.1359/JBMR.040118

31. Iannolo G, Sciuto MR, Buccheri S, et al. Numb Expression Contributes to the Maintenance of an Undifferentiated State in Human Epidermis. Cell Transplant. 2016;25(2):353-64. doi:10.3727/096368915X688245

32. Logan CY, Nusse R. The Wnt signaling pathway in development and disease. Annu Rev Cell Dev Biol. 2004;20:781-810. doi:10.1146/annurev.cellbio.20.010403.113126

33. Borello U, Buffa V, Sonnino C, Melchionna R, Vivarelli E, Cossu G. Differential expression of the Wnt putative receptors Frizzled during mouse somitogenesis. Mech Dev. Dec 1999;89(1-2):173-7.

34. Ang SJ, Stump RJ, Lovicu FJ, McAvoy JW. Spatial and temporal expression of Wnt and Dickkopf genes during murine lens development. Gene Expr Patterns. May 2004;4(3):289-95. doi:10.1016/j.modgep.2003.11.002

35. Han B, Chen SY, Zhu YT, Tseng SC. Integration of BMP/Wnt signaling to control clonal growth of limbal epithelial progenitor cells by niche cells. Stem Cell Res. Mar 2014;12(2):562-73. doi:10.1016/j.scr.2014.01.003

36. Lee HJ, Wolosin JM, Chung SH. Divergent effects of Wnt/beta-catenin signaling modifiers on the preservation of human limbal epithelial progenitors according to culture condition. Sci Rep. Nov 10 2017;7(1):15241. doi:10.1038/s41598-017-15454-x 37. McMahon AP. More surprises in the Hedgehog signaling pathway. Cell. Jan 21 2000;100(2):185-8.

38. Oliver TG, Grasfeder LL, Carroll AL, et al. Transcriptional profiling of the Sonic hedgehog response: a critical role for $\mathrm{N}$-myc in proliferation of neuronal precursors. Proc Natl Acad Sci U S A. Jun 10 2003;100(12):7331-6. doi:10.1073/pnas.0832317100 39. Belloni E, Muenke M, Roessler E, et al. Identification of Sonic hedgehog as a candidate gene responsible for holoprosencephaly. Nat Genet. Nov 1996;14(3):353-6. doi:10.1038/ng1196-353 
520 40. Roessler E, Belloni E, Gaudenz K, et al. Mutations in the human Sonic Hedgehog gene cause holoprosencephaly. Nat Genet. Nov 1996;14(3):357-60. doi:10.1038/ng1196-357 41. Choi JJ, Ting CT, Trogrlic L, et al. A role for smoothened during murine lens and cornea development. PLoS One. 2014;9(9):e108037. doi:10.1371/journal.pone.0108037

524 42. Liu Y, Xu H, An M. mTORC1 regulates apoptosis and cell proliferation in pterygium 525 via targeting autophagy and FGFR3. Sci Rep. Aug 4 2017;7(1):7339. doi:10.1038/s41598526 017-07844-y

527 43. Shin YJ, Hyon JY, Choi WS, et al. Chemical injury-induced corneal opacity and 528 neovascularization reduced by rapamycin via TGF-beta1/ERK pathways regulation. Invest 529 Ophthalmol Vis Sci. Jul 2 2013;54(7):4452-8. doi:10.1167/iovs.13-11684

530 44. Zhu J, Liu Y, Pi Y, Jia L, Wang L, Huang Y. Systemic application of sphingosine 1phosphate receptor 1 immunomodulator inhibits corneal allograft rejection in mice. Acta Ophthalmol. Feb 2014;92(1):e12-21. doi:10.1111/aos.12237

533 45. Gidfar S, Milani FY, Milani BY, et al. Rapamycin Prolongs the Survival of Corneal 534 Epithelial Cells in Culture. Sci Rep. Jan 5 2017;7:40308. doi:10.1038/srep40308

535 46. Kitazawa K, Hikichi T, Nakamura T, Sotozono C, Kinoshita S, Masui S. PAX6

536 regulates human corneal epithelium cell identity. Exp Eye Res. Jan 2017;154:30-38.

537 doi:10.1016/j.exer.2016.11.005

538 47. Bystrom B, Carracedo S, Behndig A, Gullberg D, Pedrosa-Domellof F. Alpha11

539 integrin in the human cornea: importance in development and disease. Invest Ophthalmol Vis

540 Sci. Nov 2009;50(11):5044-53. doi:10.1167/iovs.08-3261

541 48. Bystrom B, Virtanen I, Rousselle P, Miyazaki K, Linden C, Pedrosa Domellof F.

542 Laminins in normal, keratoconus, bullous keratopathy and scarred human corneas. Histochem

\section{$545 \quad$ Figure legends}

546 Figure 1: Cross-sections of fetal corneas 10-11 wg (A, D, G) and $20 \mathrm{wg}(\mathrm{B}, \mathrm{E}, \mathrm{H})$ as well

548 Dlk1 (D-F) and Numb (G-I). The corneal epithelium is shown at the top and the stroma

549 below, in all photographs of all figures. Cell nuclei are labeled blue with DAPI (A-I).

550 Immunolabeling against Notch1 (A-C) was detected as streaks in the stroma and strongly

551 around the basal layers of the epithelium in all fetal and adult corneas (A-C). In the $20 \mathrm{wg}$

552 fetal corneas the labelling of the epithelium against Notch1 was slightly surpassed by the 553 strong DAPI labelling of the epithelial cell nuclei (B). Dlk1 (D-F) labelled the epithelial cells 554 and streaks in the stroma of all fetal corneas, more abundantly in the anterior region (D-E, 555 asterisk), whereas in the adult corneas labeling was only present in the epithelium (F). In all 556 fetal corneas, Abs against Numb (G-I), another inhibitor of Notch1, labelled the epithelial 
557 cells and streaks in the stroma (G-H) but stromal labelling was more abundant in the $20 \mathrm{wg}$

$558(\mathrm{H})$ than in the 10-11 wg fetal corneas $(\mathrm{G})$. Numb labeling in adult corneas was present at the 559 epithelial cells and in sporadic streaks in the stroma (I). Bars: $100 \mu \mathrm{m}$.

561 Figure 2: Cross-sections of fetal corneas 10-11 wg (A, D, G), $20 \mathrm{wg}(\mathrm{B}, \mathrm{E}, \mathrm{H})$ and normal 562 adult corneas (C, F, I) labeled with antibodies (green) against Wnt5a (A-C), Wnt7a (D-

563 F), $\boldsymbol{\beta}$-catenin (G-I). Cell nuclei are labeled blue with DAPI (A-I). The Abs against Wnt5a 564 abundantly labelled both the epithelial cells and streaks in the stroma of the fetal corneas (A-

565 B). The stromal labelling was more abundant in the $10-11 \mathrm{wg}$ (A) than in the $20 \mathrm{wg}$ fetal 566 corneas (B), in which the streaks were more profuse in the anterior region (B). In the adult 567 corneas, only the epithelium was labelled (C). Immunolabeling against Wnt7a was found in 568 the epithelial cells of all fetal samples (D-E). The stromal labeling in all fetal corneas 569 presented streaks, slightly more abundant in the $10-11 \mathrm{wg}$ (D) than in the $20 \mathrm{wg}$ fetal corneas

570 (E). In contrast, in the adult corneas, labeling was present in the epithelium but only in extremely sparse steaks in the stroma (F). $\beta$-catenin immunolabeling was present abundantly

572 in the contours of epithelial cells but only discretely in stromal streaks, in a similar pattern in 573 the 10-11 wg $(\mathrm{G})$ and $20 \mathrm{wg}$ fetal corneas $(\mathrm{H})$. In the adult corneas, labeling was present in 574 the epithelial cells more intensively in the basal region, but absent in the stroma (I). Bars: 100 $575 \mu \mathrm{m}$.

576

577 Figure 3: Cross-sections of fetal corneas 10-11 wg (A, D, G, J) and $20 \mathrm{wg}(\mathrm{B}, \mathrm{E}, \mathrm{H}, \mathrm{K})$ 578 and adult corneas (C, F, I, L) labeled with antibodies (green) against Hes1 (A-C), Gli1 579 (D-F), mTOR (G-I) and p-rpS6 (J-L). Cell nuclei are labeled blue with DAPI (A-L). Abs 580 against Hes1 (A-C) strongly immunolabeled the epithelial cells of the fetal corneas. These 581 Abs labelled streaks in the stroma more profusely in the 10-11 wg (A) than in the $20 \mathrm{wg}$ fetal 
582 corneas (B) and more intensively in the anterior stroma (B). In contrast, in the adult corneas,

583 immunolabelling was not detected (C). Immunolabeling with Abs against Gli1was present in

584 the epithelial cells in all fetal corneas (D-E), and was more intense in the basal region of the

585 epithelium of the $20 \mathrm{wg}$ fetal corneas (E). Labelling in the stroma was present in streaks in all

586 fetal corneas (D-E) but was more abundant in the $20 \mathrm{wg}$ fetal corneas (E). Immunolabelling

587 for these Abs was not observed in adult corneas (F). The Ab against mTOR abundantly

588 labeled the epithelial cells of all fetal corneas (G-H) but marked the epithelium in the $20 \mathrm{wg}$

589 fetal corneas more intensively $(\mathrm{H})$. The stroma in all fetal corneas was labelled in streaks (G-

$590 \mathrm{H})$ but was more abundantly marked in the 10-11 wg fetal corneas $(\mathrm{G})$. This Ab did not

591 immunolabel the adult corneas (I). The Ab against p-rpS6 labeled the epithelial cells and

592 abundant streaks in the stroma of all fetal corneas in a likewise pattern in both 10-11 wg (J)

593 and $20 \mathrm{wg}$ fetal corneas (K). The stroma in the adult corneas was not labeled and the surface

594 of the epithelium was only scarcely labelled, suggesting sticky adherence to the epithelial

595 surface (L). Bars: $100 \mu \mathrm{m}$.

596

597 Figure 4: Gene expression of Notch1 (NOTCH1) (A), Wnt5A (WNT5A), Wnt7A 598 (WNT7A), $\beta$-catenin (CTNNB1) (B), and Hes1 (HES1), mTOR (mTOR), rps6 (RPS6)

599 (C) in 9-12 wg fetal corneas as compared to adult cornea. 3.5 fold increase in Notch1 gene 600 expression was detected in 9-12 wg fetal corneas. (A). Expression of Wnt5A, Wnt7A and $\beta$ 601 catenin genes was increased by 1.7, 3.75 and 2.14 fold, respectively. (B). Gene expression of 602 Hes1, mTOR and rps6 was increased by 2.2, 2.5 and 1.74 fold respectively (C). Values are 603 means \pm SD. $* * * * p<0.0001$

604

605 Tables

606 Table 1. Primary Antibodies 


\begin{tabular}{|llll|}
\hline \multicolumn{1}{|c}{ Antigen } & \multicolumn{1}{c|}{ Antibody } & Concentration & \multicolumn{1}{c|}{ Source } \\
\hline Dlk1 & Ab21682 & $1: 1000$ & Abcam, Cambridge, UK \\
Notch1 & Ab52627 & $1: 300$ & Abcam, Cambridge, UK \\
Numb & Ab14140 & $1: 250$ & Abcam, Cambridge, UK \\
Wnt5a & Ab32572 & $1: 100$ & Abcam, Cambridge, UK \\
Wnt7a & Bs-6645R & $1: 100$ & Bioss Inc, Woburn, MA, USA \\
B-catenin & Ab32572 & $1: 100$ & Abcam, Cambridge, UK \\
Hes1 & GTX 108356 & $1: 500$ & Genetex, Irvine, CA, USA \\
Gli1 & Ab 92611 & $1: 800$ & Abcam, Cambridge, UK \\
mTOR & 7C10) 2983 & $1: 500$ & Cell Signaling, Danvers, MA, USA \\
p-rpS6 & GTX 60800 & $1: 800$ & Genetex, Irvine, CA, USA \\
\hline
\end{tabular}

607

608

609 Table 2. Secondary Antibodies

\begin{tabular}{|llll|}
\hline \multicolumn{1}{|c}{ Antigen } & Antibody & \multicolumn{1}{c|}{ Concentration } & \multicolumn{1}{c|}{ Source } \\
\hline $\begin{array}{l}\text { Donkey anti-mouse } \\
\begin{array}{l}\text { DyLight 488 } \\
\text { Goat anti-mouse }\end{array}\end{array}$ & A215-485-150 & $1: 100$ & Jackson ImmunoResearch Europe Ltd, Suffolk, UK \\
$\begin{array}{l}\text { Alexa 488 } \\
\begin{array}{l}\text { Donkey anti-rabbit } \\
\text { FITC }\end{array}\end{array}$ & $1: 300$ & $\begin{array}{l}\text { Molecular Probes, Life Technologies, Darmstadt, } \\
\text { Germany }\end{array}$ \\
$\begin{array}{l}\text { Goat anti-rabbit } \\
\text { Alexa 488 }\end{array}$ & A11034 & $1: 300$ & $\begin{array}{l}\text { Jackson ImmunoResearch Europe Ltd, Suffolk, UK } \\
\text { Molecular Probes, Life Technologies, Darmstadt, }\end{array}$ \\
\hline
\end{tabular}

610

611

612

613

614

615

616

617

618

619

620 


\section{$621 \quad$ Figures}

622 Figure 1.

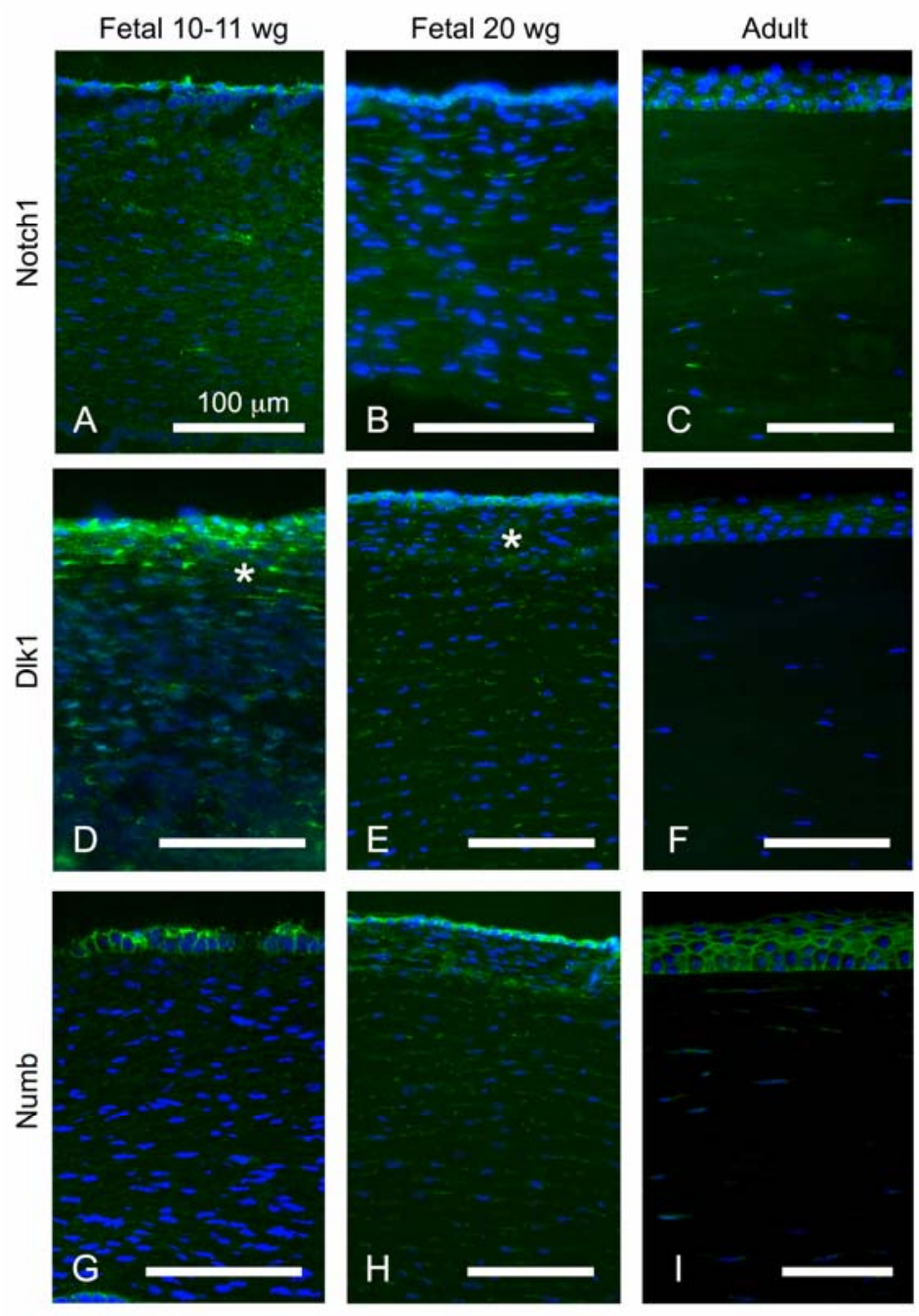

623

624

625

626

627

628

629

630

631 
bioRxiv preprint doi: https://doi.org/10.1101/2021.03.19.436141; this version posted March 19, 2021. The copyright holder for this preprint (which was not certified by peer review) is the author/funder. All rights reserved. No reuse allowed without permission.

632 Figure 2.
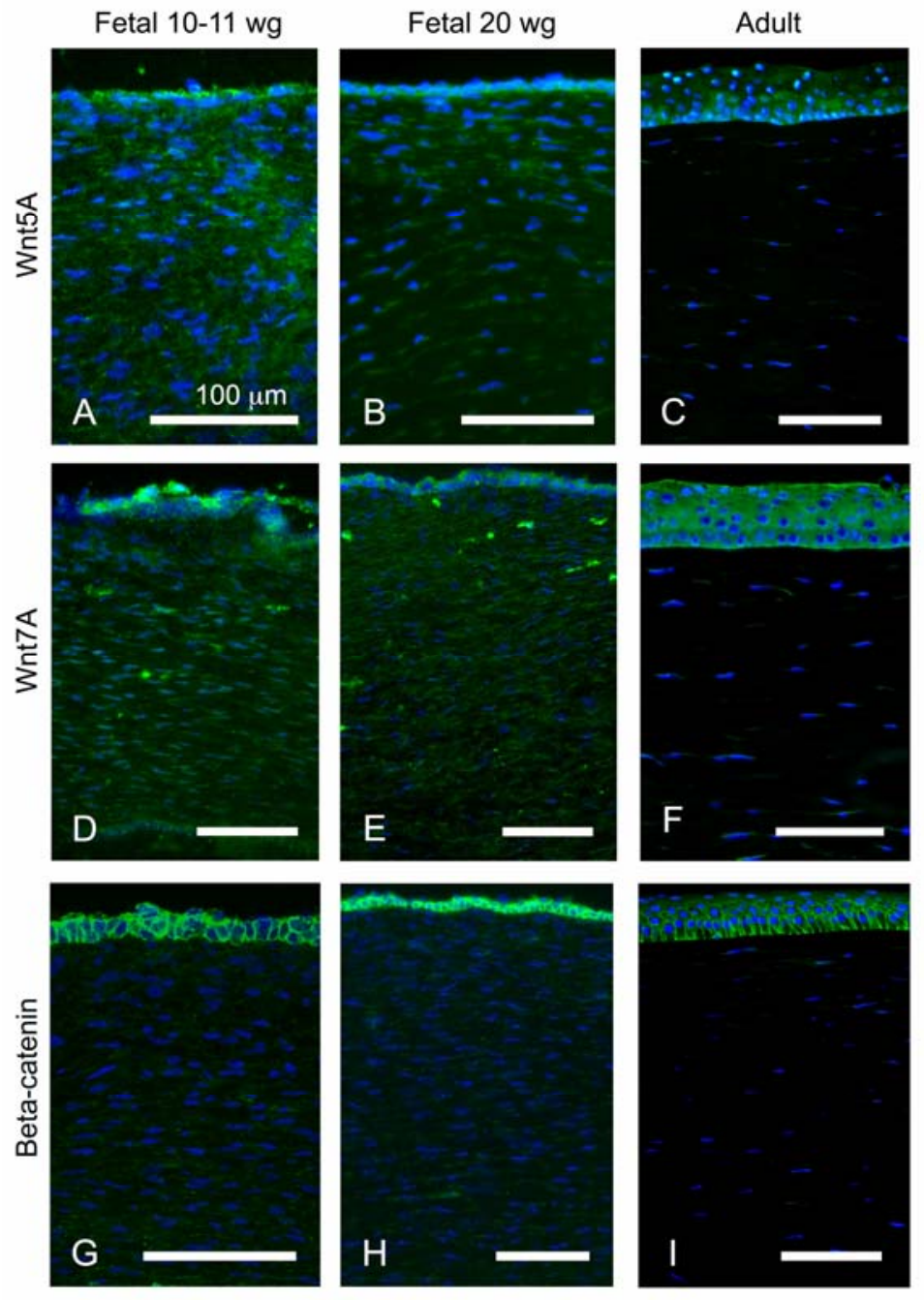

633

634

635

636

637

638

639

640

641

642 


\section{$643 \quad$ Figure 3.}



Fetal $20 \mathrm{wg}$
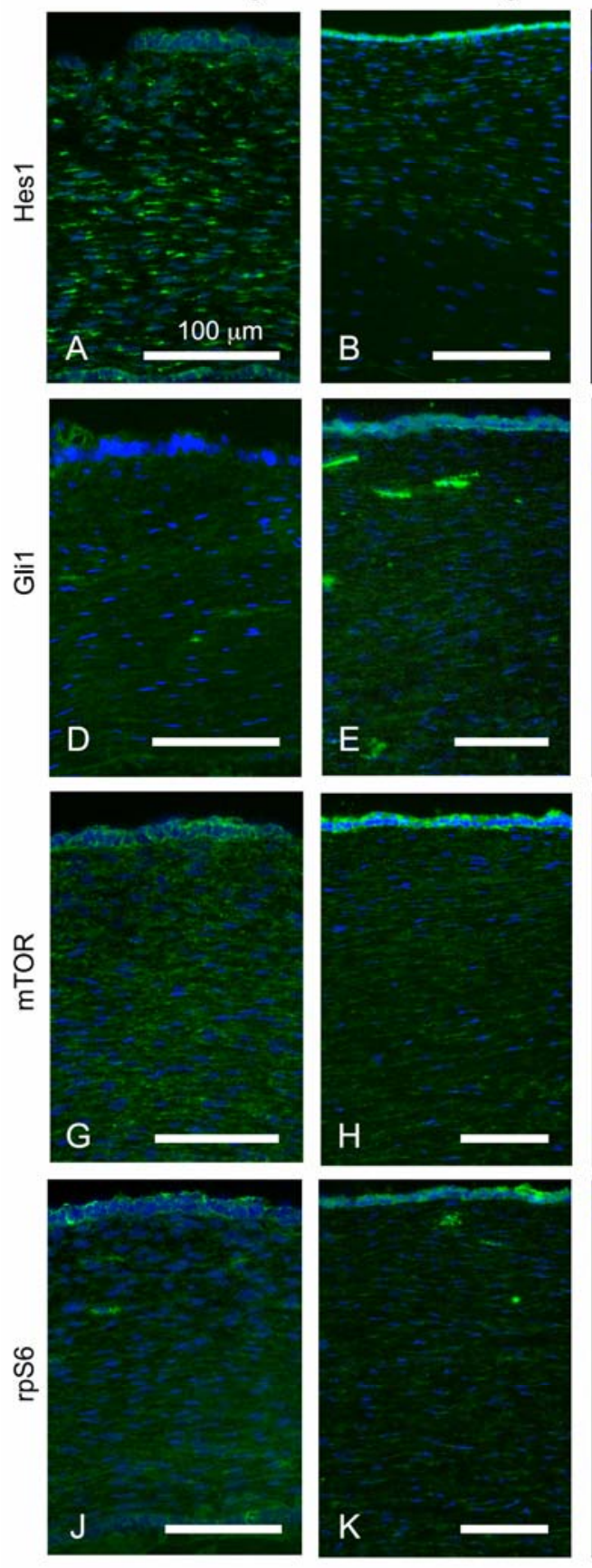



644


645

646

647

648 
bioRxiv preprint doi: https://doi.org/10.1101/2021.03.19.436141; this version posted March 19, 2021. The copyright holder for this preprint (which was not certified by peer review) is the author/funder. All rights reserved. No reuse allowed without permission.

$649 \quad$ Figure 4.

A

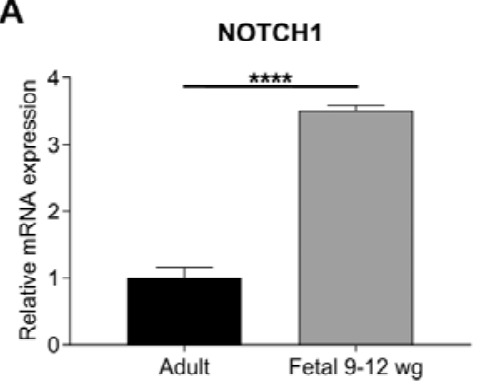

B

WNT5A

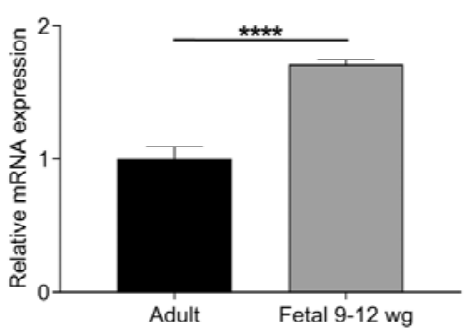

WNT7A

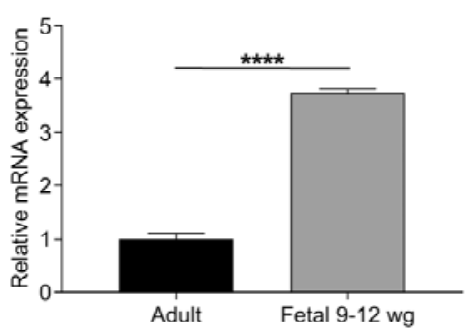

C
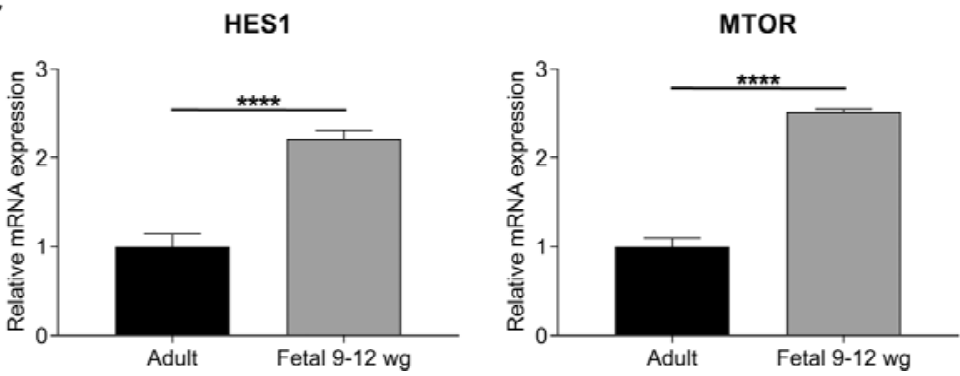

CTNNB1
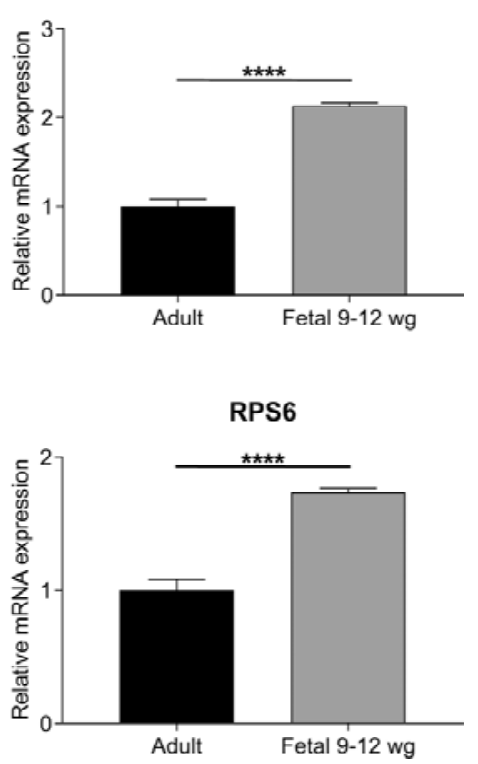